\title{
Neuroprotective Effects of Delta-9-Tetrahydrocannabinol in a Marmoset Parkinson Model
}

\author{
Sanneke A. van Vliet ${ }^{*}, 1,2$, Raymond A. Vanwersch ${ }^{1}$, Marjan J. Jongsma ${ }^{1}$, Jan van der Gugten ${ }^{2}$, \\ Berend Olivier $^{2,3}$ and Ingrid H. Philippens ${ }^{1}$
}

\author{
${ }^{I}$ Department of Diagnosis and Therapy, TNO Defence, Security and Safety, Rijswijk, The Netherlands \\ ${ }^{2}$ Department of Psychopharmacology, Utrecht Institute of Pharmaceutical Sciences and Rudolf Magnus Institute of Neu- \\ rosciences, Utrecht University, Utrecht, The Netherlands \\ ${ }^{3}$ Department of Psychiatry, Yale University School of Medicine, New Haven, USA
}

\begin{abstract}
The present medication in Parkinson's disease (PD) is unable to stop or slow down the progression of the disease. Therefore pharmacological intervention at crucial steps in the neuronal cell death processes would be a better strategy. Cannabinoids are potent neuroprotective compounds in models of oxidative stress and excitotoxicity and offer potential protection in models of PD. Therefore the present study determines the neuroprotective effects of $\Delta^{9}$ tetrahydrocannabinol $\left(\Delta^{9}\right.$-THC) in the marmoset 1-methyl-4-phenyl-1,2,3,6-tetrahydropyridine (MPTP) model on behavior and pathology. Twelve marmoset monkeys were treated with a total cumulative dose of $6 \mathrm{mg} / \mathrm{kg}$ MPTP in 9 days. Seven of these animals received simultaneously a daily oral dose of $\Delta^{9}$-THC (4 mg/kg) and five animals received simultaneously vehicle for 27 days. The parkinsonian symptoms were observed daily and locomotor activity and hand-eye coordination were tested once a week during the experimental period. Postmortem, dopamine levels in the striatum were analyzed and tyrosine hydroxylase immunohistochemistry was applied to determine viable dopaminergic neurons in the substantia nigra. $\Delta^{9}$-THC has no protective effects on any parameter. These negative results might be related to the severity of the cell death induction by MPTP in relation to the low dose of $\Delta^{9}$-THC used in this Parkinson model.
\end{abstract}

\section{INTRODUCTION}

In Parkinson's disease (PD) the output of the basal ganglia is irreversibly affected by degeneration of the neuromelanin-containing dopaminergic neurons in the substantia nigra pars compacta (SNpc). This results in symptoms including akinesia, postural instability, rigidity and resting tremors [1].

$\mathrm{PD}$ is incurable, and present medications (predominantly levodopa) do not counteract progression of the disease, and long-term medication is associated with declined efficacy and increased side-effects [2]. Therefore, a better strategy aims to focus on prevention of the neuronal loss in an attempt to stop or slow down the progression of the disease. One way to achieve neuroprotection is via pharmacological interference aimed at crucial steps in the neuronal cell death process to promote neuronal survival. Although some potential drug candidates were tested in clinical trials there is no proven neuroprotective treatment yet [2].

The actual cause of PD is unknown. There is evidence suggesting that factors like mitochondrial dysfunction, oxidative stress, excitotoxicity and inflammatory processes, either separately or cooperatively, are involved in the underlying neurodegenerative process [3].

Cannabinoids appeared neuroprotective in cerebral ischemia [4], brain trauma [5], multiple sclerosis [6] and nerve gas-induced seizures [7], but also in vivo and in vitro

*Address correspondence to this author at the Department of Diagnosis and Therapy, TNO Defence, Security and Safety, 2288 GJ Rijswijk, The Netherlands; Tel: +31 651299652; Fax: +31 152843963;

E-mail: s.a.m.van.vliet@med.umcg.nl or Sanneke.vanvliet@xs4all.nl models of oxidative stress and excitotoxicity (reviewed by [8]). Most of these protectant effects appear mediated by activation of the cannabinoid $\mathrm{CB}_{1}$ receptor [9], although the contribution of other mechanisms (i.e., anti-oxidant and/or anti-inflammatory properties of cannabinoids) have also been reported [8].

$\Delta^{9}$-tetrahydrocannabinol $\left(\Delta^{9}-\mathrm{THC}\right)$, the main component of marijuana, induces psychoactive properties via agonistic actions on cannabinoid $\mathrm{CB}_{1}$ receptors. $\Delta^{9}$-THC has neuroprotective properties in cellular and animal models of oxidative stress, ischemia, ouabain -a sodium pump blocker- induced excitotoxicity or excitotoxicity [10-16]. $\Delta^{9}$-THC administration resulted in delay of motor deterioration in an animal model of amyotrophic lateral sclerosis [17] and $\Delta^{9}$ THC partially protected against MDMA (3,4methylenedioxy-N-methylamphetamine) induced serotonin depletion [18]. These findings indicate $\Delta^{9}$-THC as a potent anti-oxidant and anti-excitotoxitant and suggest its use as a neuroprotectant in PD. Recently, the group of LastresBecker [19] showed that $\Delta^{9}$-THC protects against unilateral infusion of 6-hydroxydopamine (6-OHDA) induced neurodegeneration of the $\mathrm{SNpc}$ neurons.

To extend the investigation towards application of $\Delta^{9}$ THC in the protection of the SN neurons in PD, we tested the neuroprotective effects of $\Delta^{9}$-THC in the 1-methyl-4phenyl-1,2,3,6-tetrahydropyridine (MPTP) marmoset model for PD. MPTP, a neurotoxic agent, selectively damages dopaminergic (DAergic) neurons in the substantia nigra by blocking the electron transport chain of the mitochondria leading to a loss in mitochondrial function resulting in a depletion of ATP and finally cell death [20]. In experimental PD research the MPTP animal model is widely accepted and 
findings are confirmed in human PD brains [20,21]. The non-human primate model is preferable as these animals show after MPTP treatment clear and lasting behavioral features, which reflect many aspects of human Parkinson symptoms [21]. Even a clinically used observational scale for involuntary movements (AIMS) can be applied to the marmoset without adaptation [22]. Furthermore, similarities to humans with regard to the anatomy of the striatum, distribution of dopamine cells in the SNpc and striatal dopamine function are supportive of the choice of the non-human primate model [23].

In the present study, the protective effects of $\Delta^{9}$-THC are measured with various markers including motor functions by behavioral tests and pathological measurements at brain level. These markers are sensitive to reflect neurodegeneration and neuroprotection in the MPTP marmoset model [24]. The parkinsonian symptoms are assessed by using two extensive behavioral observation scales for PD and functional tests measuring locomotor activity and hand-eye coordination (HEC) $[25,26]$. The survival of the dopaminergic neurons in the SNpc is investigated with tyrosine hydroxylase immunohistochemistry (TH-IR). Staining of $\mathrm{TH}$, the first and rate-limiting enzyme in the synthesis of the catecholamines, is often used as a quick and sensitive measure for localization of surviving dopaminergic neurons in the MPTP-model [27]. The functionality of the surviving neurons was assessed by measurement of the level of dopamine neurotransmission in the striatum.

\section{METHODS}

\section{Animals}

Adult male and female marmoset monkeys (Callithrix jacchus), aged 2-6 years with initial body weights between 350-550 g were obtained from the primate center BPRC, The Netherlands and Harlan, United Kingdom. The ambient temperature in the housing room was regulated at $25 \pm 2{ }^{\circ} \mathrm{C}$ and the relative humidity was always $>60 \%$. A 12-h light-dark cycle was maintained, lights on from 7 am to $7 \mathrm{pm}$. All aspects of animal care are described in Standard Operating Procedures, which are in agreement with current guidelines of the European Communities Counsel Directive (86/609/EEC).

\section{Study Design}

Twelve naïve marmosets were treated cumulatively in total with $6 \mathrm{mg} / \mathrm{kg}$ MPTP s.c. over 9 days $(2 \mathrm{mg} / \mathrm{kg}$ at day 1 and $1 \mathrm{mg} / \mathrm{kg}$ at days 2, 3, 6 and 9). Seven of these animals (4 males; 3 females) additionally received a daily oral dose of 4 $\mathrm{mg} / \mathrm{kg} \Delta^{9}$-THC from experimental day 1 until day 27 . The remaining five animals ( 3 males; 2 females) additionally received a daily oral dose of the vehicle (10\% sucrose solution in water). $\Delta^{9}$-THC or vehicle treatment was given directly after the MPTP injections.

The dose of $\Delta^{9}$-THC chosen was based on a commonly used oral dose of $\Delta^{9}$-THC in non-human primates, the pharmacokinetics of oral $\Delta^{9}$-THC $[28,29,30]$ and behavioral studies at our laboratory (data not shown). $\Delta^{9}$-THC was dissolved in $50 \mathrm{mg} / \mathrm{ml}$ ethanol (IBL, Leiden, The Netherlands) and orally administered simultaneously with $1 \mathrm{ml} / \mathrm{kg} \quad 10 \%$ sucrose solution.
Before disease induction, animals were trained on the HEC task and baseline values of all test systems were obtained. The occurrence of parkinsonian symptoms was observed daily before administration of the treatment using two rating scales: clinical score and AIMS. At day 13, 20 and 27, before daily administration of the treatment, the HEC task and locomotor activity were tested in non-invasive test systems. At day 37 , ten days after the last $\Delta^{9}$-THC administration, brains were removed after decapitation under isoflurane anesthesia. One hemisphere was used for immunohistochemistry and the other for neurochemical measurements.

\section{Behavioral Assessment}

\section{Observation of Signs and Symptoms}

For the observation of signs and symptoms two rating scales were used. 1) A general clinical scoring list in which the condition of the animal is rated. The following symptoms were registered: appetite, inadequacy of grooming by inspection of the fur; apathy by testing the responsiveness of the animal to its surrounding; immobility; rigidity and presence of tremors. The rates of severity were coded from 0 (normal) to 4 (severe). 2) The AIMS is a 9-item rating scale, designed to record in detail the occurrence of involuntary movements [31]. The AIMS is widely used in the clinic for qualification of involuntary movements, occurring in PD [32]. These scales have successfully been applied for more than 10 years in monkey research in our institute. The AIMS includes facial, mouth (lips, peri-oral area, jaw and tongue), extremity and trunk movements. The global judgment of the severity and the incapacitation due to the abnormal movements were also scored. All items were rated from 0 (normal) to 4 (severe). Movements that occur upon stimulation by the observer were rated one step lower than those observed spontaneously.

\section{Spontaneous Exploratory Behavior (Bungalow Test)}

The levels of activity and exploratory behavior can play an important role in practically all measurements of animal behavior. A device called the 'Bungalow test' automatically and quantitatively assesses these parameters and is extensively described and validated [25,26]. The apparatus consists of four horizontally placed non-transparent boxes $(23 \mathrm{x}$ $23 \times 23 \mathrm{~cm}$ ) all interconnected by 6 PVC tubes (inner diameter $9.5 \mathrm{~cm})$. Each animal was placed in the same compartment at the start of each session. The animals could freely move and change from one compartment to another during the 20-min session. A video tracking system (Ethovision, Noldus bv., Wageningen, The Netherlands) registered the locomotor activity of the animal, expressed as the number of compartment changes during the session.

\section{Hand-Eye Coordination Task}

An automated robot-guided apparatus with positive reinforcement as a motivating stimulus (small pieces of marshmallow) has been used to asses the HEC [26]. The marmoset is placed in front of a test panel provided with a window $(8 \mathrm{x}$ $5 \mathrm{~cm}$ ). A robot arm presents a reward behind the window. With this system three types of trials were performed: one using a non-moving reward in the middle of the window, one using a slow horizontally moving reward $(0.04 \mathrm{~m} / \mathrm{s})$ and one using a fast horizontally moving reward $(0.08 \mathrm{~m} / \mathrm{s})$. The animal was allowed one minute to grasp the non-moving 
reward. Each type of trial was presented 14 times in one session. At the beginning of each trial a sound signal was presented, intended to alert the animal. A pressure detector in the robot arm and infrared detectors in the window registered hits and attempts and speed of performance. A 'hit' was registered when the animal successfully retrieved the reward from the robot arm. The percentage of correct hits was used as a criterion to judge the performance of the animal.

\section{HPLC Analysis}

For determination of brain dopamine levels, five brains of the $\Delta^{9}$-THC treated PD-group and five from the vehicle treated PD- group were used. Furthermore, six brains of naivve animals were used to establish control values of the dopamine. At day 37, ten days after the last $\Delta^{9}$-THC administration, brains were removed after decapitation of the sedated animals. The striatum of one hemisphere was isolated after termination and was directly frozen in liquid nitrogen. The tissue (100-500 mg) was weighed and homogenized in $10 \mathrm{ml}$ $0.4 \mathrm{M}$ perchloric acid containing $20 \mathrm{ng} / \mathrm{ml} 3,4-$ dihydroxybenzylamine hydrobromide (Sigma chemical Co. St. Louis, MO, USA) and $20 \mathrm{ng} / \mathrm{ml}( \pm)$-isoproterenol hemisulfate salt (Sigma chemical Co. St. Louis, MO, USA) as internal standards. Homogenate was centrifuged at 22,000 $\mathrm{g}$ for 30 minutes at $4^{\circ} \mathrm{C}$ and $1 \mathrm{ml}$ of supernatant was adjusted to about $\mathrm{pH} 4.0$ with $250 \mu \mathrm{l} 2 \mathrm{M}$ sodium acetate. The homogenate samples were stored at $-70^{\circ} \mathrm{C}$ for a maximum of 6 weeks. Dopamine and the metabolites 3,4dihydroxyphenylacetic acid (DOPAC), 3-methoxy-4hydroxyphenylacetic acid (HVA) were determined by ionpair reversed phase liquid chromatography. A 2-50 $\mu 1$ sample was injected on a RP18 LiChrosfer 100 column (125 x 4 mm i.d., $5 \mu \mathrm{m}$ particle size; Merck, Darmstadt, Germany) connected to a Thermo Separations Products (San Jose, CA, USA) model P100 solvent delivery pump and AS300 autosampler and a Coulochem II Model 5011 electrochemical detector (ESA, Bedford, MA, USA). The mobile phase consisted of a $30 \mathrm{mM}$ citrate $/ 40 \mathrm{mM}$ phosphate buffer, $\mathrm{pH} 4.0$, containing $0.27 \mathrm{mM} \mathrm{Na} \mathrm{F}_{2}$ EDA, $1.8 \mathrm{mM}$ heptanesulphonic acid and 5\% methanol. The potential of the electrode was set at $420 \mathrm{mV}$. External standards were determined in each assay run. Calibration plots were linear from 2 to $500 \mathrm{ng} / \mathrm{ml}$ for each compound. The lower limit of detection was $2 \mathrm{ng} / \mathrm{ml}$. The intra-assay coefficient of variation amounted to $2 \%$.

\section{Immunohistochemistry}

Brains (vehicle treated-PD group: $n=5 ; \Delta^{9}$-THC treatedPD group: $n=5$ ) were analyzed for the presence of dopaminergic neurons with tyrosine hydroxylase immunoreactivity. Four brains of naïve animals (control group) were used to establish control values. From collected brains, the right hemisphere was fixated in $4 \%$ paraformaldehyde at $4^{\circ} \mathrm{C}$ and after 48 hours the brains were transferred to $0.5 \%$ paraformaldehyde at $4^{\circ} \mathrm{C}$. Brains were dehydrated in graded ethanol and xylene and subsequently embedded in paraffin. Serial transversal sections of $5 \mu \mathrm{m}$ were cut on a microtome and collected serially on aminosilane/acetone solution coated slides. Every $4^{\text {th }}$ section was mounted on egg-white coated glass slides for cresylviolet staining which was used as reference for the TH-IR localization.

The sections used for the TH-IR were deparaffinated and rehydrated in xylene and graded ethanol. The citrate buffer method was applied for antigen retrieval. Sections were preincubated with $0.3 \% \mathrm{H}_{2} \mathrm{O}_{2}$ in $\mathrm{PBS}$ to quench endogenous peroxidase activity. Thereafter the sections were preincubated in PBS with $0.1 \%$ bovine serum albumin (BSA) and $0.3 \%$ Triton $\mathrm{X}-100$. Incubation in anti-TH serum (1:80000, Sigma chemical Co. St. Louis, MO, USA) was overnight at room temperature. The secondary antibody (1:2000, Santa Cruz Biotechnology inc., CA, USA) was incubated for 90 minutes followed by a 90 -minute incubation with Vector ABC (1:800, Vector laboratories inc., Burlingame, Canada). PBS-washes were applied after each pre- and antibody incubation step. Thereafter the sections were pre-incubated for 10 minutes with $0.025 \% 3^{\prime} 3^{\prime}$ diaminobenzidine containing $0.15 \%$ nickel ammonium sulphate (DAB-NI solution) followed by a 10-minute incubation in the DAB-NI solution with $0.00015 \% \mathrm{H}_{2} \mathrm{O}_{2}$ to visualize bound immunocomplexes. After a PBS-wash the sections were dehydrated in alcohol series, cleared up in xylol and cover-slipped with DePeX (BDH Laboratory supplies, England).

TH-IR positive neurons were counted in 4 sections of the SNpc A4- $5.5 \mathrm{~mm}$ anterior of the external auditory meati (marmoset brain atlas: Stephan et al. 1980). Within each section, TH-IR neurons were counted manually in three medial to lateral parts of the SNpc using an eye-piece grid of 10 $\mathrm{mm} \times 10 \mathrm{~mm}$ at a magnification of 400x on a Olympus light microscope.

\section{Statistics}

The results of this study are presented as mean \pm SEM and parametric statistical analysis was applied with a significance level of $p<0.05$. The scores of the behavioral observation scales were analyzed with an independent t-test to reveal differences between the two treatment groups. The results of the behavioral tests were analyzed in two ways. First, the difference between the two treatment groups was obtained. Therefore an overall repeated measure analysis was applied on the results of day 13, 20 and 27. When relevant, an independent t-test was applied. Second, the difference between baseline and test day results of each treatment values was tested with a paired t-test.

The difference between the dopamine levels of each treatment was tested with a one-way ANOVA followed by a t-test when relevant. Immunochemical data were evaluated with a one-way ANOVA followed by multiple comparison procedure (Bonferroni corrected t-test).

\section{RESULTS}

\section{Parkinsonian Symptoms}

The behavior rated with the observation scales showed that $\Delta^{9}$-THC could not prevent the development of parkinsonian symptoms during MPTP intoxication as both groups had comparable observational scores (Fig. 1). During the first three MPTP injections the $\Delta^{9}$-THC treated PD-animals were even worse than the vehicle treated PD-animals. Significant differences were found with the AIMS at day 2 and 3 and with the CS at day $5(\mathrm{p}<0.05)$.

Similar to the findings during observations, the HEC performance (Fig. 2) and locomotor activity (Fig. 3), tested during day 13, 20 and 27, were lower than before disease induc- 
tion in both treatment groups $(\mathrm{p}<0.05)$. Moreover, no differences between these two groups were found in the test systems.

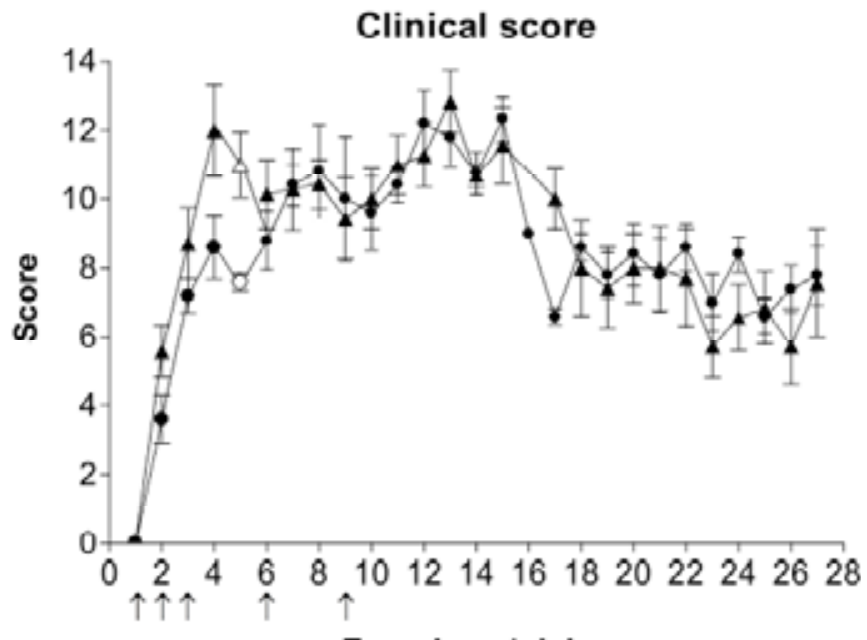

Experimental day

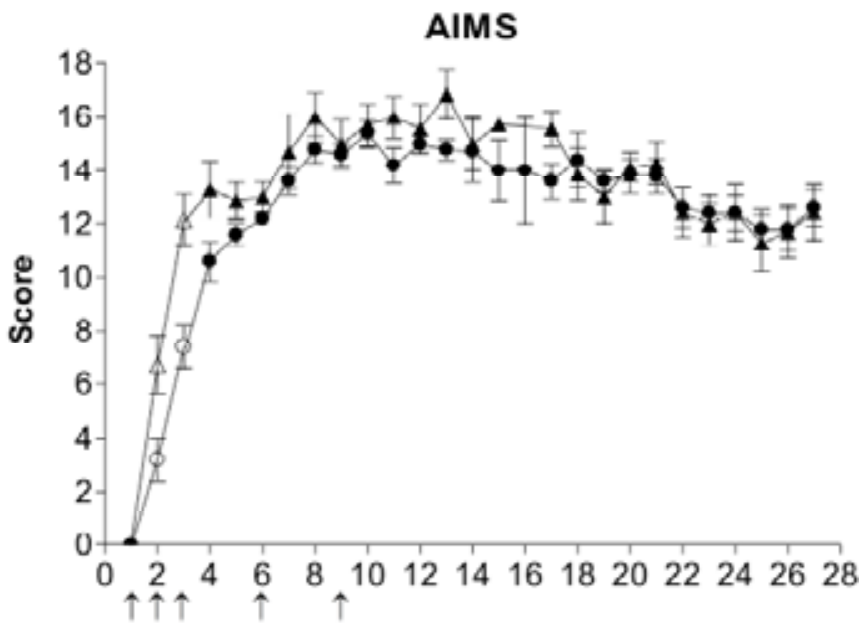

Experimental day

Fig. (1). Mean \pm SEM of the rating scales before daily administration. Circles indicate vehicle treated PD-animals and triangles $\Delta^{9}$ THC treated PD-animals. Arrows indicate days of MPTP injection. $\Delta^{9}$-THC or vehicle was given from day 1 to 27 . Open data points : $\mathrm{p}<0.05$ between the two treatment groups.

\section{Biochemical Analysis}

The levels of DA and its metabolites, HVA and DOPAC, were clearly reduced in the MPTP + vehicle and MPTP $+\Delta^{9}$ THC group $(\mathrm{p}<0.001$, Table 1). No differences were found between both groups.

\section{Immunohistochemistry}

Also at cellular level the inability of $\Delta^{9}$-THC to protect against the MPTP intoxication was clear (see images in Fig. (4)). Both MPTP groups had a similar number of remaining DAergic neurons. In the MPTP + vehicle group a reduction of $75.8 \pm 5.3 \%$ of TH-IR neurons was found $(\mathrm{p}<0.001 \mathrm{vs}$ control) and in the $\Delta^{9}$-THC treated MPTP group a reduction of $70.7 \pm 4.2 \%$ of number of TH-IR neurons was found ( $\mathrm{p}<0.001$ vs control).

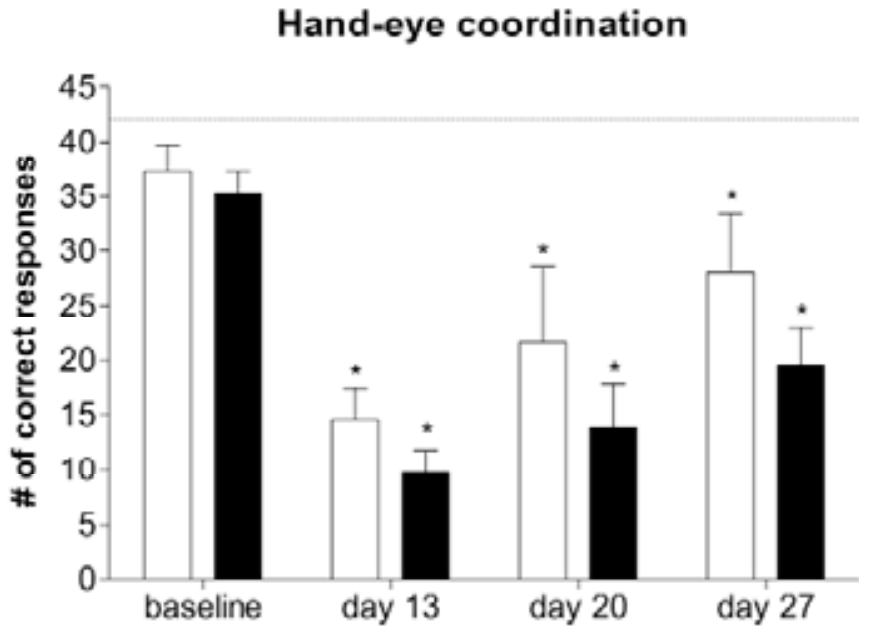

Fig. (2). Mean + SEM of performance in the HEC task of the vehicle treated PD-animals (white bars) and $\Delta^{9}$-THC treated PDanimals (black bars). Dashed line indicates maximum number of reward presentations. $* \mathrm{p}<0.05$ vs baseline values.

\section{Locomotor activity}

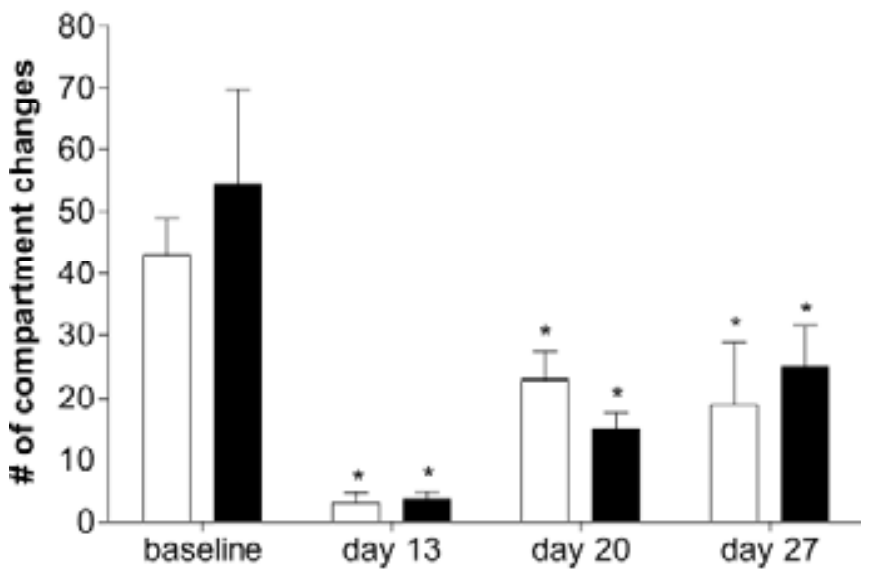

Fig. (3). Mean + SEM of the locomotor activity in the Bungalow test of the vehicle treated PD-animals (white bars) and $\Delta^{9}$-THC treated PD-animals (black bars). $* \mathrm{p}<0.05$ vs baseline values. No difference between baseline values.

\section{DISCUSSION}

This study is one of the first investigating the neuroprotective effects of $\Delta^{9}$-THC in a non-human primate model of Parkinson's disease. Recently, the group of Lastres-Becker [19] reported positive results with $\Delta^{9}$-THC, a cannabinoid $\mathrm{CB}_{1}$ receptor agonist, in the unilateral 6-OHDA rat model. In that study $3 \mathrm{mg} / \mathrm{kg} \Delta^{9}$-THC i.p. was given 16 hours after the 6-OHDA infusion and continued daily for 2 weeks. In contrast to the findings in the 6-OHDA model, orally given $\Delta^{9}$ THC in a dose of $4 \mathrm{mg} / \mathrm{kg}$ was not able to protect the DAergic neurons in the SNpc against MPTP induced neurodegeneration in the marmoset monkey. The parkinsonian symptoms were not reduced after $\Delta^{9}$-THC treatment, damage of the DAergic neurons was not prevented and the functionality of the surviving neurons was comparable to vehicle treatment. 
Table 1. Dopamine and Metabolite Levels (Mean \pm SEM) in $\mu \mathrm{g} / \mathrm{g}$ Tissue in the Striatum of the Vehicle $(\mathrm{n}=5)$ and $\Delta$-THC $(n=5)$ Treated PD-Animals Compared to Untreated Control $(n=6)$ Levels

\begin{tabular}{|c|c|c|c|}
\hline & Control & MPTP + Vehicle & MPTP + THC \\
\hline \hline DA & $5.47 \pm 0.85$ & $0.27 \pm 0.08 * * *$ & $0.47 \pm 0.17 * *$ \\
DOPAC & $0.73 \pm 0.06$ & $0.16 \pm 0.06 * * *$ & $0.28 \pm 0.02 * * *$ \\
HVA & $5.37 \pm 0.59$ & $0.34 \pm 0.09 * * *$ & $0.66 \pm 0.15 * * *$ \\
DA turnover & $1.24 \pm 0.18$ & $2.10 \pm 0.29 *$ & $3.50 \pm 1.28$ \\
\hline
\end{tabular}

DA turnover: ((DOPAC $+\mathrm{HVA}) / \mathrm{DA})$; $* * * v s$ control levels $\mathrm{p}<0.001 ; * * v s$ control levels $\mathrm{p}<0.01 ; * v s$ control levels $\mathrm{p}<0.05$ (ANOVA followed by t-test).

The MPTP model has proven to be useful for studying neuroprotective effects. The behavioral markers used for parkinsonian symptoms, DA levels and neuronal survival, are sensitive to neurodegeneration by MPTP and neuroprotection in the marmoset monkey [19]. Therefore, the cause of the discrepancy between the results of the 6-OHDA rat study and this study can be due to the differences between the experimental designs, which will be discussed in the following sections.

The application of two different neurotoxins could be an explanation for the discrepancy. However, the mechanisms of 6-OHDA and MPTP intoxication imply comparable cellular modifications susceptible to induce cell death of DAergic cells [33]. Both methods result in mitochondrial deficits and oxidative stress, although the induction of oxidative stress is slightly different as 6-OHDA generates reactive oxygen species (ROS) via deamination by monoamine oxidase-B or auto-oxidation and 1-methyl-4-phenylpyridinium $\left(\mathrm{MPP}^{+}\right)$, the effective metabolite of MPTP, becomes a radical after reaction with xanthine oxidase [33]. ROS and radicals can both be deactivated with anti-oxidants [3]. This implicates that cell death processes induced via MPTP or 6-OHDA would be comparably susceptible for neuroprotective intervention with cannabinoids.

Despite the comparable cellular modification, the time frame of cell death differs between the models. Degeneration starts 12 hours after a single MPTP dose and continues till day 4 post-treatment [34], whereas after a single dose of 6OHDA degeneration starts 12 hours post-injection and ends 7-10 days later [35].

Furthermore, in the present study MPTP was given with repeated injections, which is a normal procedure in the MPTP non-human primate model. Therefore, the severity of DA depletion in this study is higher than in the 6-OHDA study of Lastres-Becker [19] (95\% vs 46\% reduction). The severity level of cell death might be of primary importance in the success of neuroprotective compounds to prevent cell death. Therefore, the more severe induction scheme used in the present study might need a different strategy in the application of the neuroprotective compound, e.g., different doses.

The dose of the compound is essential for the success of a neuroprotective compound. Oral administration of $4 \mathrm{mg} / \mathrm{kg}$ $\Delta^{9}$-THC used in this study was a pharmacological effective dose as this dose evoked behavioral responses in naïve marmoset monkeys (data not published). This dose did is
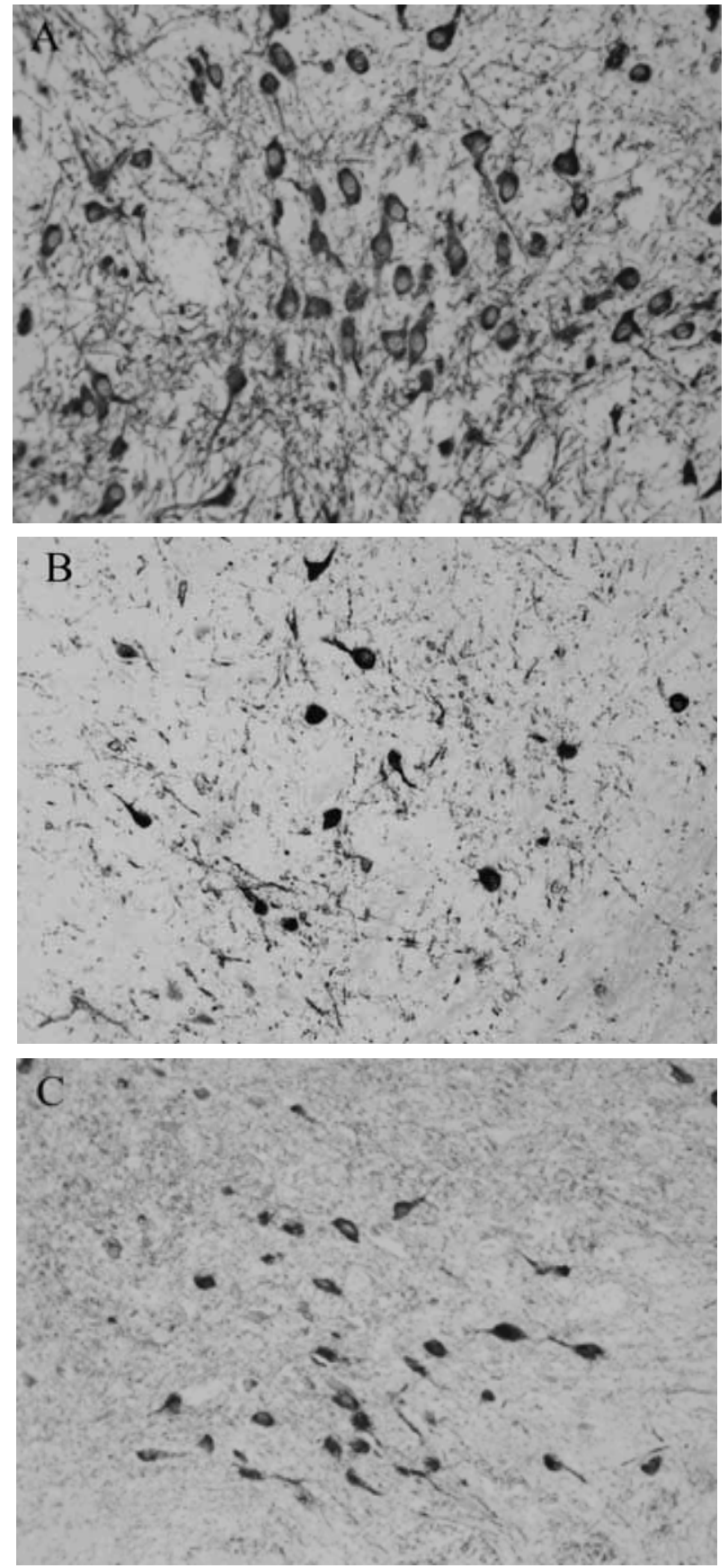

Fig. (4). Images of the TH-IR neurons in the SNpc (magnification $200 \mathrm{x}$ ) of a control animal (A), $\Delta^{9}$-THC treated (B) and vehicle treated $(\mathbf{C})$ PD-animal.

comparable with the dose of $3 \mathrm{mg} / \mathrm{kg}$ used in the 6-OHDA model, although the method of administration can affect the dose available for the neuroprotection processes. In this study, $\Delta^{9}$-THC was orally administered as the plasma concentration of $\Delta^{9}$-THC is more stable over time in contrast to inhalation and could therefore generate a longer lasting neuroprotective effect. After oral intake, the level of $\Delta^{9}$-THC in human plasma is maximal $60-120$ minutes post-dosing and has an elimination half-life of 25 hours [30]. The low clearance level after oral intake may benefit the neuroprotective effects of $\Delta^{9}$-THC as the degeneration process takes a couple of days [34]. A disadvantage of oral administration is the low bioavailability $50 \%$ lower than after inhalation or rectal 
administration) due to degradation by the acid of the stomach, the gut and the first-pass effect [30]. I.p. injections shortcut these effects and the total bioavailability is expected to be higher as well as the peak concentration. Therefore an assumption may be that in the present MPTP experiment the $\Delta^{9}$-THC concentration in the brain to exert neuroprotective actions was clearly lower than after i.p. injections in the 6OHDA experiment [19].

The importance of the right concentration of cannabinoids in the brain during neuroprotection is illustrated by the need of a higher concentration for anti-oxidative than receptor-mediated protection [8]. The anti-oxidant properties of cannabinoids responsible for the protection of DAergic neurons are further consolidated with a recent demonstration in the 6-OHDA model [36]. It revealed that cannabinoids which acted independently of the cannabinoid $\mathrm{CB}_{1}$ receptor were neuroprotective and synthetic cannabinoid $\mathrm{CB}_{1}$ agonists failed to be neuroprotective. $\Delta^{9}$-THC has, besides its agonistic actions on the cannabinoid $\mathrm{CB}_{1}$ receptors, also antioxidant properties as shown in oxidative stress models [10]. If the protection of the DAergic neurons is indeed not mediated via cannabinoid $\mathrm{CB}_{1}$ receptors, a too low $\Delta^{9}$-THC dose may contribute to the explanation of failure in preventing DAergic neuronal death after MPTP. The dose used in this study was high enough to stimulate the cannabinoid $\mathrm{CB}_{1}$ receptor as this dose generated typical behavioral effects like apathy and bradykinesia in marmosets at our institute (data not published). This supports the finding of Garcia-Arencibia [36] that the neuroprotective effect of $\Delta^{9}$-THC is not regulated by direct action on the cannabinoid $\mathrm{CB}_{1}$ receptor.

In conclusion, in this study oral $\Delta^{9}$-THC in a dose of 4 $\mathrm{mg} / \mathrm{kg}$ did not prevent severe DAergic cell death after repeated MPTP injections. These results contrast to the findings in the 6-OHDA model [19]. The underlying explanation could be a combination of a more severe damage model and a rather low dose for anti-oxidant protection. Studies with a higher dose of $\Delta^{9}$-THC and administration before disease induction to create a steady state level of $\Delta^{9}$-THC is needed to examine whether the neuroprotective potential of $\Delta^{9}$-THC is mediated via its anti-oxidant properties or to refute the existence of neuroprotection by $\Delta^{9}$-THC in a PD model close to man.

\section{ACKNOWLEDGEMENT}

We want to thank Wim van der Wal for his technical assistance during the HPLC analysis.

\section{REFERENCES}

[1] Fearnley, J.M.; Lees, A.J. Brain, 1991, 114, 2238-2301.

[2] Clarke, C.E. Lancet Neurol., 2004, 3, 466-74.

[3] Alexi, T.; Borlongan, C.V.; Faull, R.L.; Williams, C.E.; Clark, R.G.; Gluckman, P.D.; Hughes, P.E. Prog. Neurobiol., 2000, 60, 409-70.

[4] Nagayama, T.; Sinor, A.D.; Simon, R.P.; Chen, J.; Graham, S.H.; Jin, K.; Greenberg, D.A. J. Neurosci., 1999, 19, 2987-95.
[5] Panikashvili, D.; Simeonidou, C.; Ben-Shabat, S.; Hanus, L.; Breuer, A.; Mechoulam, R.; Shohami, E. Nature, 2001, 413, 527531.

[6] Lyman, W.D.; Abrams, G.A.; Raine, C.S. J. Neuroimmunol., 1989, 25, 195-201.

[7] Filbert, M.G.; Forster, J.S.; Smith, C.D.; Ballough, G.P. Ann. $N Y$ Acad. Sci., 1999, 890, 505-14.

[8] Grundy, R.I.; Rabuffetti, M.; Beltramo, M. Mol. Neurobiol., 2001, 24, 29-51.

[9] Parmentier-Batteur, S.; Jin, K.; Mao, X.O.; Xie, L.; Greenberg, D.A. J. Neurosci., 2002, 22, 9771-5.

[10] Hampson, A.J.; Grimaldi, M.; Axelrod, J.; Wink, D. Proc. Natl. Acad. Sci. USA, 1998, 95, 8268-73.

[11] van der Stelt, M.; Veldhuis, W.B.; Bar, P.R.; Veldink, G.A.; Vliegenthart, J.F.; Nicolay, K. J. Neurosci., 2001, 21, 6475-9.

[12] El Remessy, A.B.; Khalil, I.E.; Matragoon, S.; Abou-Mohamed, G.; Tsai, N.J.; Roon, P.; Caldwell, R.B.; Caldwell, R.W.; Green, K.; Liou, G.I. Am. J. Pathol., 2003, 163, 1997-2008.

[13] Chen, J.; Lee, C.T.; Errico, S.; Deng, X.; Cadet, J.L.; Freed, W.J. Brain Res. Mol. Brain Res., 2005, 134, 215-225.

[14] Chen, J.; Errico, S.L.; Freed, W.J. Neurosci. Lett., 2005, 389, 99103.

[15] Hayakawa, K.; Mishima, K.; Nozako, M.; Ogata, A.; Hazekawa, M.; Liu, A.X.; Fujioka, M.; Abe, K.; Hasebe, N.; Egashira, N.; Iwasaki, K.; Fujiwara, M. Neuropharmacol., 2007, 52, 1079-87.

[16] Gilbert, G.L.; Kim, H.J.; Waataja, J.J.; Thayer, S.A. Brain Res., 2007, 1128, 61-9.

[17] Raman, C.; McAllister, S.D.; Rizvi, G.; Patel, S.G.; Moore, D.H.; Abood, M.E. Amyotroph Lateral Scler Other Motor Neuron Disord., 2004, 5, 33-39.

[18] Morley, K.C.; Li, K.M.; Hunt, G.E.; Mallet, P.E.; McGregor, I.S. Neuropharmacol., 2004, 46, 954-965.

[19] Lastres-Becker, I.; Molina-Holgado, F.; Ramos, J.A.; Mechoulam, R.; Fernandez-Ruiz, J. Neurobiol. Dis., 2005, 19, 96-107.

[20] Dauer, W.; Przedborski, S. Neuron, 2003, 39, 889-909.

[21] Jenner, P.; Marsden, C.D. J. Neural. Transm., 1986, 20, 11-39.

[22] Di Monte, D.A.; McCormack, A.; Petzinger, G.; Janson, A.M.; Quik, M.; Langston, W.J. Mov. Disord., 2000, 15, 459-66.

[23] Eslamboli, A. Brain Res. Bull., 2005, 68, 140-9.

[24] van Vliet, S.A.; Vanwersch, R.A.; Jongsma, M.J.; van der Gugten, J.; Olivier, B.; Philippens, I.H. Behav. Pharmacol., 2006, 17, 45362 .

[25] Wolthuis, O.L.; Groen, B.; Philippens, I.H. Pharmacol. Biochem. Behav., 1994, 47, 879-881.

[26] Philippens, I.H.; Melchers, B.P.; Roeling, T.A.; Bruijnzeel, P.L. Behav. Res. Methods Instrum. Comput., 2000, 32, 173-179.

[27] Pearson, J.; Goldstein, M.; Markey, K.; Brandeis, L. Neuroscience, 1983, 8, 3-32.

[28] Perlin, E.; Smith, C.G.; Nichols, A.I.; Almirez, R.; Flora, K.P.; Cradock, J.C.; Peck, C.C. J. Pharm. Sci., 1985, 74, 171-4.

[29] Aigner, T.G. Psychopharmacol., (Berl) 1988, 95, 507-11.

[30] Grotenhermen, F. Clin. Pharmacokinet., 2003, 42, 327-60.

[31] Guy, W. ECDEU assessment manual for psychopharmacology. Washington D.C: U.S. Department of Health, Education and Welfare; 2007.

[32] Katzenschlager, R.; Manson, A.J.; Evans, A.; Watt, H.; Lees, A.J. J. Neurol. Neurosurg. Psychiatry, 2004, 75, 295-7.

[33] Blum, D.; Torch, S.; Lambeng, N.; Nissou, M.; Benabid, A.L.; Sadoul, R.; Verna, J.M. Prog. Neurobiol., 2001, 65, 135-72.

[34] Jackson-Lewis, V.; Jakowec, M.; Burke, R.E.; Przedborski, S. Neurodegeneration, 1995, 4, 257-69.

[35] Jeon, B.S.; Jackson-Lewis, V.; Burke, R.E. Neurodegeneration, 1995, 4, 131-7.

[36] Garcia-Arencibia, M.; Gonzalez, S.; de Lago, E.; Ramos, J.A.; Mechoulam, R.; Fernandez-Ruiz, J. Brain Res., 2007, 1134, 162170. 